\title{
COVID-19, the Pandemic, and Challenges Associated with Vaccines
}

\author{
Cheryl Ann Alexander'and Lidong Wang2* \\ 'Institute for IT innovation and Smart Health, Mississippi, USA \\ ${ }^{2}$ Institute for Systems Engineering Research, Mississippi State University, Mississippi, USA \\ *Corresponding author: Lidong Wang, Institute for Systems Engineering Research, Mississippi State University, Mississippi, \\ USA
}

\section{Abstract}

The COVID-19 pandemic has brought many issues facing the international community as a whole to the forefront. Issues related to achieving herd immunity, to wear a mask or not to mask, get the vaccine or skip the vaccine, as well as the numerous issues of the pandemic that surround our children during the pandemic and issues surrounding vaccination. Vaccines have been a major concern during the COVID-19 pandemic. This paper introduces the types of methods, techniques, and platforms involved in making COVID-19 vaccines; vaccines against COVID-19 and the length of time between first and second doses; the efficacy of vaccines; the variants or mutations and the efficacy of vaccines against the variants; the side effects of vaccines; vaccines for individuals with complications or pregnancy; herd immunity; and vaccine hesitancy.

Keywords: COVID-19, SARS-CoV-2, vaccines, variants, mutations, herd immunity, vaccine hesitancy

\section{Introduction}

Nature has a powerful way of reminding us of her ability to produce severe infectious disease which can sicken, kill, and disrupt even the most economically and technologically advanced societies. When Pandora's Box was opened in December 2019, multiple international governments immediately started breaking down the genomic sequence of SARS-CoV-2 to discover and potentially develop therapeutics and vaccines. President Donald J. Trump, USA, began Operation Warp Speed (OWS) in February 2020, when the first cases were hitting the US. Operation Warp Speed gathered numerous pharmaceutical corporations, research and development organizations, and the US government to attempt what had never been done prior to this pandemic; it was the goal of Operation Warp Speed to produce a viable vaccine to combat the pandemic, while simultaneously producing millions of doses of vaccines for populations most vulnerable to COVID-19. Therefore, when it was announced in mid-November that two mRNA vaccines had received emergency use authorizations (EUA) by the Food and Drug Administration (FDA) in the USA. BNT162b2 (Pfizer and BioNTech) and Moderna (ModernaTX, Inc, USA), showed around $95 \%$ efficacy for preventing symptomatic
COVID-19 and about 100\% efficacy in preventing severe COVID-19 or hospitalizations in clinical trials. One study showed a $94.5 \%-95 \%$ reduction in asymptomatic infections 14 or more days after the second dose of the Pfizer/ BioNTech vaccine or the Moderna vaccine. Today it is wellknown that all vaccines require at least a 14-day window for the vaccine to establish a viable antibody response and provide established immunity for the person vaccinated [1].

The FDA issued an EUA on February 27, 2021, for the third vaccine developed within OWS, a vaccine developed by Johnson \& Johnson using a recombinant, replicationincompetent adenovirus serotype 26 vector vaccine, encoding the stabilized prefusion spike glycoprotein of SARS-CoV-2 [2]. The Johnson \& Johnson Janssen COVID-19 vaccine unfortunately caused a scare for patients desiring the one-shot vaccination as multiple individuals suffered and some died as a result of rare blood clots in the large abdominal vessels, the cerebral venous sinuses, and other sites, and the large vessels of the lower extremities. This thrombosis with thrombocytopenia shared clinical course features with the autoimmune heparin-induced thrombocytopenia following vaccination. The FDA did at one point, halt the administration of the J\&J Janssen vaccine, but 
it has since been resumed (J\&J Janssen COVID-19 vaccine @ janssencovid19vaccine.com). By January 2021, 30 vaccines had entered the human trial phases and 165 vaccines had registered for trials. Additional entries were seen including Medigen from Taiwan, ReiThera from Italy, and ZydusCadila's Indian subsidiary. Other countries, such as China, Russia, and others were cooperating with other countries in Central America and Western Europe for approval. Some vaccines were in the market that had received EUA by the FDA or the European Medicines Agency (EMA), such as Moderna's mRNA-based vaccine, BioNtech-Pfizer joint venture vaccine, the Johnson \& Johnson's Janssen vaccine, and the Oxford-AstraZeneca's ChAdOx [3].

The objective behind the push for vaccination began almost with the development of the first vaccines. Herd immunity, an epidemiological term that means most of the population of any given populace has reached immunity, either naturally by infection with the pathogen or through an acquired immunity gained through vaccination, has always been the goal of the international community affected by COVID-19. Some countries were on target to reach herd immunity even without the vaccines as the infection rates during the peak of COVID-19 had reached over $70 \%$, which would have pushed the population over the numbers necessary for herd immunity. However, in the US, for example, some politicians and pundits, as well as some key pandemic practitioners kept changing the percentages of the population necessary to reach herd immunity, in some cases to extend the pandemic for financial and political gain. Other countries, such as Italy, began the push for vaccination as it was the first European country to make COVID-19 vaccination mandatory for healthcare workers. Serbia also considered making a similar policy. In Ireland, the Health Information and Quality Authority advised adoption of a policy that encouraged "progressive" interventions to persuade healthcare workers who refused to take vaccines [4]. In March 2021, fears of vaccine shortage led to the Indian government temporarily stopping exports of the University of Oxford-AstraZeneca vaccine (Covishield) produced by the Serum Institute of India in Pune [5]. COVID-19 vaccine donation by the US to low- and middleincome countries was investigated but became a political issue. For example, in the US, older Americans were less likely to endorse vaccine donations and were more likely to want all Americans eligible for vaccination to have access. On the other hand, Democrats favored vaccine donation more so than Republicans, who supported vaccination of all eligible Americans first [6].

During the heights of the COVID-19 pandemic, many people chose or were forced to work from home, doctors conducted telemedicine appointments, and many states stayed on lockdown for several months, causing employers to question how to bring employees back to the workplace, customers back to malls, theatres, and restaurants and bars, etc. Government and medical officials juggled with various ideas on how the US could safely assure a person was not contagious, or had received the vaccine, making them safe to return to work. In response, a COVID-19 'Immunity Passport' was promoted as one approach to enabling activities to resume and some cities and states have established mandates requiring these 'immunity passports' to engage in work, go to theatres, dine in a restaurant, drink at a bar, etc. Hence, mobile phone app has been developed based on the need for a COVID-19 Antibody Test/Vaccination Certificate, verifiable credentials, the distributed storage of cryptographic public/key pairs, and the decentralized verification of data with confidentiality [7]. Since many international countries have been developing COVID-19 vaccines, and the question of vaccine efficacy has become the most important question, determinants such as vaccine features or place of vaccination has been investigated. Although most determinants are hypothetical, analyses are especially significant in a country where vaccine confidence is continually low [8].

The purpose of this paper is to discuss and expose significant issues regarding COVID-19 vaccines. This paper is organized as follows: introduction of primary issues, the second section introduces types of methods, techniques, and platforms involved in making vaccines; the third section presents vaccines against COVID-19 and length of time between first and second doses; the fourth section introduces the efficacy of vaccines; the fifth section presents variants or mutations and efficacy of vaccines against the variants; the sixth section introduces side effects of vaccines; the seventh section deals with vaccines for individuals with complications or pregnancy; the eighth introduces herd immunity; the nineth section discusses vaccine hesitancy; and the tenth section is a conclusion.

\section{Spike Protein and Challenges in Vaccine Production}

For the last 70 years, vaccine production has primarily relied on an adenovirus or vector virus grown in chicken eggs to establish immunity. Most seasonal-flu vaccines produced in the US still use this method. Production of vaccines in eggs using a vector virus is a somewhat slow process and can take weeks. Vector virus vaccines also require specific pathogen-free chicken eggs and takes up too much space since each egg only yields one dose of vaccine. Eggs are still a mainstay in vaccine production; however, newer, faster, and more efficient methods using mammalian cells are stepping up. Researchers have turned to mammalian cells to make

Citation: Cheryl Ann Alexander1and Lidong Wang2* COVID-19, the Pandemic, and Challenges Associated with Vaccines

Op Acc J Bio Sci \& Res 9(1)-2021 
more viral vaccine quickly in a more controlled environment and in much less space than the egg-based approach [9].

Our understanding of the SARS-CoV-2 virus is primarily based on what limited studies are available on other members of the coronavirus family, which has been circulating since 2003. Therefore, characteristics of the immune response to an infection with other members of the coronavirus family should serve as the theoretical basis for targeting the antigen for SARS-CoV-2. SARS-CoV-2 has four major structural proteins: spike (S), membrane (M), envelope (E), and nucleocapsid (N). The $S$ protein is used as the target antigen in vaccine development as it is responsible for recognizing the host receptor which allows the virus to enter the cell. Although the $\mathrm{N}$ protein is highly immunogenic and plays the vital role in coronavirus infections of packaging the viral RNA into a helical capsid essential for the viability of the virus. It is virtually impossible to detail the N protein's complex role in inducing the immunological response as a target for neutralizing and protecting the host against the virus, as studies have revealed that $\mathrm{N}$ antibodies are not neutralizing nor protecting. Further examination of the $\mathrm{N}$ protein, as well as various other viral antigens which do show the positive stimulation of the host immune system by different antigen components of SARS-CoV-2. This confirmed presence of antibodies in the convalescent sera of COVID-19 patients from multiple proteins and components of the virus, raises the question about how SARS-CoV-2 releases antibodies against multiple components throughout the disease course. Therefore, researchers need to consider the presence of other viral antigens, particularly the $\mathrm{N}$ antigen, in addition to the $S$ antigen as a main component inducing neutralizing antibodies [10].

Many pharmaceutical companies are now considering a variety of major vaccine platforms from the traditional recombinant design to the latest biotechnology mRNA vaccines which takes the genetic footprint of the virus and creates a viable vaccine. A high level of safety is also a primary goal of pharmaceutical companies engaged in vaccine production and there is always the theory that any vaccine could also make the SARS-CoV- 2 virus much more severe through genetic alteration or arbitrary mutation of the virus. However, data from in vivo studies on what would be necessary to prevent re-infection or how long that protection would last by studying the $S$ protein, replicating and nonreplicating viral vectors, and nucleic acid DNA and mRNA. Significant factors include safety and reactogenicity, speed and flexibility of manufacture, vaccine stability, durability of immunity, cold chain requirements, scale and cost of manufacturing, and the profile of humoral and cellular immunogenicity. Some companies have developed nucleic acid-based vaccines, including Moderna, BioNTech/ Pfizer, Inovio (DNA-based), and CureVac (mRNA-based). DNA- and mRNA-based vaccines may be produced quickly based on the viral sequence. mRNA vaccines utilize lipid nanoparticles to protect and deliver the mRNA and elicit the immunogenic response effectively. The scalability of the lipid nanoparticles and the temperature stability should be resolved as soon as possible to increase the ease for transportation and storage of the mRNA vaccines [11].

A prominent feature of the vaccine development for COVID-19 is the range of technology platforms being assessed, including nucleic acid (DNA and RNA), viral vector (replicating and non-replicating), recombinant protein, peptide, virus-like particles, live attenuated virus, and inactivated virus methods. Some of the platforms are currently not the basis for licensed vaccines. Four main platforms for vaccines against COVID-19 are of nucleic acid vaccines, virus vaccines, viral vector vaccines, and proteinbased vaccines. Vaccines based on viral vectors present a high- level of protein expression and long-term stability and induce greater immune responses $[12,13]$.

Three kinds of COVID-19 vaccines in the market have been tested in adult clinical trials. They are: 1 ) messenger RNA (mRNA) vaccines; 2 ) vector vaccines; and 3) protein subunit vaccines. It also takes at least two weeks for the human body to build immunity after vaccination. mRNA vaccines have genetic elements specific to SARS-CoV-2. After the genetic vaccine is administered, the host's cells begin to produce the protein antigen that mimics the virus. In turn, the body makes antibodies, T-Cells, and B-lymphocytes to combat the protein, constructing memory cells and protection for future exposure to the virus. The protein subunits of the vaccines have harmless proteins of SARSCoV-2 [14]. The authorized COVID-19 vaccines currently available utilize various approaches to establish immunity such as messenger RNA (mRNA), viral vector, DNA vaccine, protein subunit, and virus inactivated immunizations [15]. Table 1 explains the primary features of the RNA vaccine, the protein vaccine, the viral vector vaccine, and the inactivated-virus vaccine [16].

\section{Vaccines against COVID-19 and Length of Time between First and Second Doses}

Vaccines approved or used in multiple countries are designed to block the spike protein located on the SARSCoV-2 virus. Involved in a variety of cellular functions, the $S$ protein functions primarily as the cell receptor which enables the virus to infect the human host cell; acts in determining the virulence of the COVID-19 virus, among other functions. Vaccines that have been approved by the 
Table 1: Methods used in making COVID-19 vaccines.

\begin{tabular}{|c|c|}
\hline Ways & Description \\
\hline mRNA vaccine & $\begin{array}{l}\text { - A segment of coronavirus RNA coding to produce spike proteins is synthesized. } \\
\text { - The RNA segment is combined with fatty acids and cholesterol to produce lipid delivery particles. } \\
\text { - The particles are fused with membranes of human cells which release the RNA. These cells also make spike } \\
\text { proteins (triggering an immune response). }\end{array}$ \\
\hline Protein vaccine & $\begin{array}{l}\text { - An insect virus is engineered to contain coronavirus genetic material. } \\
\text { - The engineered virus grows in the insect cell environment and makes coronavirus spike proteins. } \\
\text { - The proteins are combined with harmless microscopic particles. } \\
\text { - The particles take the proteins into the body for triggering an immune response. }\end{array}$ \\
\hline Viral vector vaccine & $\begin{array}{l}\text { - A harmless adenovirus is engineered to contain coronavirus genetic material. } \\
\text { - The engineered virus grows in the cell environment. } \\
\text { - The virus is separated from cells and purified into a vaccine. } \\
\text { - The adenovirus cannot replicate inside human cells; however, it makes coronavirus spike proteins (triggering } \\
\text { an immune response). }\end{array}$ \\
\hline $\begin{array}{l}\text { Inactivated-virus } \\
\text { vaccine }\end{array}$ & $\begin{array}{l}\text { - A live coronavirus is permitted to infect trillions of mammalian cells in a bioreactor. } \\
\text { - The virus is separated from the cells and chemically treated to inactivate its RNA. } \\
\text { - The inactivated virus cannot replicate inside human cells; however, its intact proteins can trigger immune } \\
\text { responses. }\end{array}$ \\
\hline
\end{tabular}

Table 2: Some popular COVID-19 vaccines.

\begin{tabular}{|c|c|c|}
\hline $\begin{array}{c}\text { Name of the vaccine } \\
\text { or its company }\end{array}$ & Country & Types of the vaccine \\
\hline Moderna & USA & $\begin{array}{l}\text { RNA vaccine, nucleic } \\
\text { acid vaccine }\end{array}$ \\
\hline Pfizer/BioNTech & $\begin{array}{c}\text { USA/ } \\
\text { Germany }\end{array}$ & $\begin{array}{l}\text { RNA vaccine, nucleic } \\
\text { acid vaccine }\end{array}$ \\
\hline Janssen & USA & $\begin{array}{l}\text { Viral vector vaccine, } \\
\text { nucleic acid vaccine }\end{array}$ \\
\hline Oxford-AztraZeneca & UK & $\begin{array}{l}\text { Viral vector vaccine, } \\
\text { nucleic acid vaccine }\end{array}$ \\
\hline Gamaleya-Sputnik V & Russia & $\begin{array}{l}\text { Viral vector vaccine, } \\
\text { nucleic acid vaccine }\end{array}$ \\
\hline $\begin{array}{l}\text { Medicago (Plant } \\
\text { based vaccine) }\end{array}$ & Canada & $\begin{array}{l}\text { Viral subunit vaccine, } \\
\text { protein vaccine }\end{array}$ \\
\hline Sanofi & France & $\begin{array}{c}\text { Viral subunit vaccine, } \\
\text { protein vaccine }\end{array}$ \\
\hline CanSino biologics & China & $\begin{array}{l}\text { Viral vector vaccine, } \\
\text { nucleic acid vaccine }\end{array}$ \\
\hline SinoPharm & China & $\begin{array}{c}\text { Inactivated vaccine, viral } \\
\text { vaccine }\end{array}$ \\
\hline Bharat Biotech & India & $\begin{array}{c}\text { Inactivated vaccine, viral } \\
\text { vaccine }\end{array}$ \\
\hline
\end{tabular}

FDA or EMA are: BNT162b2, mRNA-1273 (Moderna, USA), AZD1222 (Oxford-AstraZeneca), Janssen COVID-19 Vaccine (Johnson \& Johnson, USA). Sputnik V (Gamaleya, Russia). BBIBP-CorV (SinoPharm, China), and CoronaVac (Sinovac, China) have been used in some Central American countries and others [17].

COVID-19 vaccines include genetic vaccines-DNA and RNA vaccines, viral vector vaccines, viral subunit vaccines, and inactivated virus vaccines. DNA vaccines are made up of small strands of DNA, a gene, encoding the Spike. RNA vaccines consist of a strand of mRNA, which encodes the antigen, or S-Protein. Viral vector vaccines use a harmless virus known as a vector to carry a foreign gene, for example, the S-Protein. As for viral subunit vaccines, subunits of the pathogen are used in vaccine preparation. These vaccines can produce strong antibody responses. Inactivated vaccines contain a whole pathogen that is inactivated by chemicals, heat, or radiation [18]. Some COVID-19 vaccines, their types, and production counties are shown in Table 2 [18]. Various jurisdictions allowed for a prolonged second dosing interval. Table 3 [19] shows some recommendations for mRNA COVID-19 vaccines from different regulating bodies.

\section{Efficacy of Vaccines}

Reference [20] gave the calculation of vaccine efficacy as vaccine efficacy $(\%)=(1-O R) \times 100$, where the OR is called

Citation: Cheryl Ann Alexander1and Lidong Wang2* COVID-19, the Pandemic, and Challenges Associated with Vaccines Op Acc J Bio Sci \& Res 9(1)-2021 
odds ratio and is calculated as:

$$
\mathrm{OR}=((\mathrm{a} / \mathrm{b})) /(\mathrm{c} / \mathrm{d})=((\mathrm{a} \times \mathrm{d})) /((\mathrm{b} \times \mathrm{c})))
$$

where $a$ is the number of vaccinated participants infected with COVID-19, $b$ is the number of vaccinated participants without the infection of COVID-19, c is the number of unvaccinated participants infected with COVID-19, and $d$ is the number of unvaccinated participants without the infection of COVID-19.

Reference [21] presented two kinds of calculation for vaccine efficacies: 1) (1-(risk among vaccinated patients/ risk among unvaccinated patients) $) \times 100$, based on those remaining at risk according to a specified analysis period; 2 ) (1-IRR) $\times 100$, where IRR is the computed ratio of confirmed cases of COVID-19 per 1000 person-years of follow-up in the active vaccine group to the corresponding sickness rate in a placebo group.

mRNA-based Moderna and Pfizer vaccines have 9495\% efficacy in stopping symptomatic COVID-19 and the vaccine efficacy can be calculated as ( 1 - the attack rate with vaccine divided by the attack rate with placebo) $\times 100$. Specifically, in a population such as the one registered in trials, with a cumulated COVID-19 attack rate over a period of three months of about $1 \%$ without any vaccine, it is expected that approximate $0.05 \%$ of vaccinated individuals could be infected. It is the meaning that $95 \%$ of individuals are secure from COVID-19 due to a vaccine [22]. Vaccines help stop viruses mainly (but not exclusively) by eliciting the neutralization of antibodies that block spike and hence thwart the ability of SARS-CoV-2 in infecting cells. Around $95 \%$ efficacy of the BNT162b2 mRNA vaccine has indicated that eliciting the neutralization of antibodies to spikes significantly related to protection from the virus due to vaccines [23].

In a study of nursing home residents who had asymptomatic COVID-19 detected through twice-weekly surveillance testing, receipt of a single dose of the BNT162b2 vaccine within the previous three weeks was related to a substantially less nasopharyngeal viral load than was detected in the absence of vaccination. An mRNA vaccine can have an instant effect on lowering the spread of SARS-CoV-2 among nursing home residents with a high risk after the first dose; the single-dose strategy may be a viable public health approach. In a randomized, observerblinded, and placebo-controlled trial, the BNT162b2 mRNA SARS-CoV-2 vaccine demonstrated $52 \%$ efficacy against symptomatic COVID-19 within the 21 days between first and second doses, and 95\% efficacy when greater than seven days after the second dose [24].
Results from a UK surveillance study showed that a reduction of COVID-19 infections by $65 \%$ after the first dose of the Oxford-AstraZeneca. No evidence indicated the difference between the Pfizer and AstraZeneca vaccines in reducing infection rates besides faintly different immune responses. Two doses of Pfizer obtained strong responses of antibody for all ages. There is less reduction in asymptomatic infection than that with symptoms, highlighting the potential for people with vaccination to be infected with SARS-CoV-2 [25].

Variants, Mutations, and the efficacy of Vaccines against the Variants

Evidence demonstrated a reduction in the numbers of asymptomatic COVID-19 among individuals who were vaccinated by a series of nasal swab testing. Variants of SARS-CoV-2 have been detected in the USA, South Africa, United Kingdom, etc. These variants share the N501Y substitution, which affects the spike (S) protein and the viral receptor binding site for cell entry. Early epidemiological evidence has shown that mRNA vaccine-induced immune responses may be effective in neutralizing the variants [15]. In March 2021, India's health ministry said gene sequencing by a consortium of ten national research laboratories had revealed that several COVID-19 variants were circulating in the country. One of the variants is B.1.1.7 variant, which was first detected in the United Kingdom, then spread more broadly to the international community [5]. This variant, also known as the Delta variant, is more effective in causing infection, even among the vaccinated. However, the Delta variant is not any more deadly than other variants. Individuals who are already vaccinated can expect to suffer a mild case of flu-like symptoms or be asymptomatic.

There was an increase in the number of viral variants in New York City in the US. Most of these variants were the B.1.526 variant first identified in New York City and the B.1.1.7 variant. Emerging SARS-CoV-2 variants have greater clinical concerns over bed shortages, rising infection and death rates, and the propensity the Delta variant has for infecting children, even the youngest infants. There have been cases of several people with vaccine breakthrough infection though they completed the second dose of mRNA1273 (Moderna) or BNT162b2 (Pfizer-BioNTech). Despite evidence of the efficacy of the vaccines, some individuals still developed COVID-19 symptoms evidenced by a positive test using the gold standard, or the polymerase-chain-reaction (PCR) testing for SARS-CoV-2, despite completion of the dual doses or single dose of the vaccine. Viral sequencing confirmed specific variants, indicating a possible risk after vaccination is completed. The ability of variants to evade vaccine-induced immunity and lead to asymptomatic or

Citation: Cheryl Ann Alexander1and Lidong Wang2* COVID-19, the Pandemic, and Challenges Associated with Vaccines Op Acc J Bio Sci \& Res 9(1)-2021 
Table 3: Recommendations regarding the second dose interval for mRNA COVID-19 vaccines from various jurisdictions.

\begin{tabular}{|c|c|c|}
\hline Organizations & $\begin{array}{l}\text { In favor of maintaining dosing as } \\
\text { recommended by manufacturers }\end{array}$ & In favor of extending the dosing interval \\
\hline WHO & & $\begin{array}{l}\text { may be extended up to } 42 \text { days based on available clinical trial } \\
\text { data }\end{array}$ \\
\hline US CDC & & $\begin{array}{l}\text { The interval is greater than } 1 \text { month for Moderna and } 3 \text { weeks } \\
\text { for Pfizer-BioNTech; no maximum interval for } \\
\text { either vaccine }\end{array}$ \\
\hline US FDA & $\begin{array}{l}\text { The second dose should be as close to the } \\
\text { recommended interval as possible, i.e., } 21 \text { days or } \\
28 \text { days. }\end{array}$ & \\
\hline Pfizer-BioNTech & $\begin{array}{l}\text { No data show that protection after the first dose is } \\
\text { sustained after } 21 \text { days. }\end{array}$ & \\
\hline $\begin{array}{l}\text { European Medicines } \\
\text { Agency }\end{array}$ & & $\begin{array}{l}\text { Maximum interval-42 days for full protection using Pfizer- } \\
\text { BioNTech }\end{array}$ \\
\hline $\begin{array}{l}\text { British Society of } \\
\text { Immunology }\end{array}$ & & $\begin{array}{l}\text { Delaying a second 'booster' dose of a protein antigen vaccine } \\
\text { (e.g., Pfizer-BioNTech and AZN) by eight weeks is not likely } \\
\text { to have any negative effect on the overall immune response } \\
\text { post-boost. }\end{array}$ \\
\hline $\begin{array}{l}\text { Government of } \\
\text { Canada }\end{array}$ & $\begin{array}{c}\text { For Moderna: } \\
\text { Minimum interval-21days, authorized } \\
\text { interval-28 days, } \\
\text { alternate interval-none. } \\
\text { For Pfizer-BioNTech: } \\
\text { Minimum interval-19 days, authorized } \\
\text { interval-21 days, alternate interval-28 days. }\end{array}$ & \\
\hline
\end{tabular}

Table 4: Common adverse effects of Moderna and Pfizer-BioNTech.

\begin{tabular}{|c|c|c|}
\hline $\begin{array}{c}\text { Adverse effects } \\
\text { or reactions }\end{array}$ & $\begin{array}{c}\text { Moderna } \\
(\%)\end{array}$ & $\begin{array}{c}\text { Pfizer-BioNTech } \\
(\%)\end{array}$ \\
\hline $\begin{array}{c}\text { Injection site reaction, } \\
\text { including pain }\end{array}$ & 92 & 84.1 \\
\hline Fatigue & 70 & 62.9 \\
\hline Headache & 64.7 & 55.1 \\
\hline Muscle pain & 61.5 & 38.3 \\
\hline Joint pain & 46.4 & 23.6 \\
\hline Chills & 45.4 & 31.9 \\
\hline Fever & 15.5 & 14.2 \\
\hline
\end{tabular}

symptomatic infection is of a major concern [26].

An important mutation in SARS-CoV-2 is the spike D614G substitution. This mutation facilitates an easier binding with the ACE2 receptor of the respiratory tract. This mutation does not increase viral pathogenesis in animal models but does enhance viral replication in the upper respiratory tract of humans, leading to more efficient transmission via respiratory aerosols. Despite this spike protein mutation, mRNA-based vaccines that encode D614 antigens have still achieved 94-95\% efficacies, which is difficult to improve on during the initial months of the pandemic [27].

The evolution of viral infections, including mutations, may involve deletions, insertions, misincorporations, or recombination, as well as natural selection for favorite features (e.g., more efficient viral transmission, replication, and the evasion of host defenses). The continuous spread of SARS-CoV-2 presents a chance for the buildup of more consequent mutations in S and throughout the viral genome. Mutations in the spike protein of a SARS-CoV-2 variant can weaken immunity. Increased capacity of phenotypic and genotypic testing is important within the global to identify and characterize a SARS-CoV-2 variant. P.1, B.1.351, and B.1.1.7 are primary spreading variants, and each has at least eight single, nonsynonymous nucleotide changes. Other variants with multiple mutations in S1 include B.1.525, A.23.1, the lineages B.1.526 (identified in New York, USA), and B.1.429 (occurred in California, USA) with a substitution in the receptor-binding domain. It was thought that B.1.525 and A.23.1 first occurred in Nigeria and Uganda, respectively [28].

Citation: Cheryl Ann Alexander1and Lidong Wang2* COVID-19, the Pandemic, and Challenges Associated with Vaccines Op Acc J Bio Sci \& Res 9(1)-2021 
A SARS-CoV-2 lineage known as B.1.1.7 spreads faster than other strains. This variant has many mutations. One of mutations, N501Y, is in the receptor binding site. The spike with the mutation binds more tightly to ACE-2 (its cellular receptor). BNT162b2-immune sera neutralized SARS-CoV-2 with an introduced N501Y mutation as effectively as they neutralized SARS-CoV-2 without a mutation. The essentially preserved neutralization of pseudo-viruses bearing the B.1.1.7 spike by BNT162b2-immune sera makes it impossible for the UK variant to escape BNT162b2-mediated protection [29]. The variant $\Delta 382$ linked to mild COVID-19 infection. The mutation named "VUI-202012/01" comprises a mutation in the coronavirus genome, leading to much more rapid spread of COVID-19. Mutations (generally deletion) in ORF8 may influence treatments, the development of vaccines, and the discovery of drugs in the future [3].

Mutations that evade the vaccine may occur when immunity wanes or because of incomplete vaccination (e.g., taking only one of two required vaccines), so complete vaccination and monitoring immune responses need to be performed [30]. Studies suggested that the emergence and spread of SARS-CoV-2 variants should be related to the lack of strong immune protection after first exposure to previous (wild-type) viruses or even to vaccines. This evolution, regarding the emergence of immune escape mutants, has not only been observed with SARS-CoV-2. Such evolution may be supported by the waning of the immune response and remarkably the antibody response. The quick arrival of variants (e.g., variants first detected in South Africa and Brazil) indicated a natural immune evasion. The dynamics of natural or vaccinal collective immunity in regions where the variants occurred may have put considerable pressure on the viral ecosystem, helping the arrival of a variant with increased transmissibility [31].

There has been a concern about decreased vaccineinduced immune protection to emergent variants with mutations in the spike protein. The variant B.1.1.7 (also named 501Y.V1) occurred in the UK in September 2020. It has eight amino acid changes in the spike. The B.1.1.7 spike mutations affect transmission as well as immune recognition. The E484K mutation was observed in the variant B.1.351 (also called 501Y.V2) that occurred in South Africa. The variant P.1 emerged in Brazil and sporadic examples from UK sequencing showed E484K on the B.1.1.7 background [23]. A study found that both Pfizer-BioNTech and OxfordAstraZeneca vaccines appeared to be very effective against infection compatible with the Kent variant (B.1.1.7) [25].

The spike protein in SARS-CoV-2 is the target of antibodies in convalescent and vaccine sera. 23 mutations in spike protein have been reported in the variants P.1, B.1.1.7, and B.1.351. Viral mutations often occur due to the instability of SARS-CoV-2 RNA and error-prone replication. Distinct from inactivated vaccines developed in China, western countries prefer to develop viral vector vaccines or mRNA vaccines, targeting the spike protein because its mutations are worthy of constant monitoring. There have been following guidance or suggestions regarding COVID-19 vaccines and therapy: 1) inactivated vaccine seed strain of spreading SARS-CoV-2 variants deserves development for future epidemics; 2) desired vaccine candidates, multivalent vaccines, or cocktail vaccines ought to be developed to neutralize all spreading variants; and 3) a personalized antibody therapy or cocktail therapy against COVID-19 should be useful through the local spreading variants screening for patients [32].

\section{Transparency and Issues Related to Vaccine Side} Effects

Safety monitoring for COVID-19 vaccines has been important. The Vaccine Adverse Event Reporting System (VAERS) that is a spontaneous reporting system and v-safe that is an active surveillance system have been used for monitoring for side effects of the vaccines in the US. VAERS is a national passive surveillance system used for adverse events after vaccination; it collects reports from the public, healthcare providers, and manufacturers of vaccines. $V$-safe is a safety monitoring system created by the US CDC, however, it has not received the public attention needed to implement mass vaccination. Headache, fatigue, myalgia, and injection site pain have been most often reported, with a greater frequency after the second dose. There are more reactions on the vaccination day than any other day [33]. Other reports of side effects include fatal or debilitating blood clots, myocarditis, heart attacks, and strokes.

European regulators expressed a concern about a potential link between rare blood clots and a vaccine developed by AstraZeneca at Cambridge and the University of Oxford in the UK. Clots tentatively linked to the AstraZeneca and J\&J vaccines have special features: occurring in uncommon parts of the body (e.g., the abdomen or brain), or being coupled with low levels of platelets, and cell fragments that facilitate blood coagulation. Early reports indicated that younger women with vaccination were the most vulnerable to blood clots; however, the European Medicines Agency suggested that it should not recognize any particular group with a high risk from the data of the AstraZeneca vaccine. The obvious bias towards women might be due to vaccination priority for healthcare workers who are primarily female in many countries [34]. Anaphylaxis to mRNA vaccines is predicted at 2.5-11.1 cases per million doses, mainly in

Citation: Cheryl Ann Alexander1and Lidong Wang2* COVID-19, the Pandemic, and Challenges Associated with Vaccines Op Acc J Bio Sci \& Res 9(1)-2021 
people with the allergy history [15]. Anaphylaxis is a lifethreatening allergic reaction that appears infrequently after vaccination. During December 14-23, 2020, monitoring by the VAERS identified 21 cases of anaphylaxis after the administration of a reported 1,893,360 first doses of the Pfizer-BioNTech vaccine; $71 \%$ of them appeared within 15 minutes after vaccination. Immediate treatment on anaphylaxis is needed. The following should be ensured: 1) healthcare providers early diagnose symptoms and signs of anaphylaxis; 2) essential supplies should be available in vaccination locations to handle anaphylaxis; 3 ) suspected anaphylaxis is treated with intramuscular epinephrine; and 4) people suffering anaphylaxis are transferred to facilities where suitable medical treatment is available for them [35].

"COVID arm" is an unusual adverse effect that may appear as a localized and transient erythematous rash several days after the first dose of Moderna. Topical steroids and oral histamines have shown success in controlling its symptoms and clearing the rash. Patients should be informed by providers that COVID arm is a benign possible side effect; therefore, the second dose of Moderna should not be stopped due to COVID arm [36]. Both Moderna and Pfizer-BioNTech may cause local and systemic adverse effects that are shown in Table 4 [37]. The effects can be mild to moderate and last 24 hours or less. Acetaminophen can alleviate these symptoms, but it cannot completely remove them.

\section{Vaccines for Individuals with Complications or Pregnancy}

The probability of the COVID-19 infection is greater in patients with multiple sclerosis who are older, have significant baseline disability, and have cardiovascular or pulmonary comorbidities. B cell depleting therapies might also raise the risk; discontinuation of these therapies for high-risk patients should be considered. The therapies (e.g., ocrelizumab and rituximab) reduce the immunization effect of vaccines although the degree of effect differs with the vaccine and timing [38]. A case report has suggested that pharmacovigilance on cardiac injury be considered, particularly with suspected or confirmed previous COVID-19 history aimed to actively search for acute myocarditis when discomfort or chest pain is reported [39].

Immunocompromised people have been excluded from the studies of mRNA vaccines of COVID-19. In these patients, the immune response to vaccination can be reduced. The humoral response to the first dose in solid organ transplant recipients was studied and quantified to better know about the immunogenicity of mRNA vaccines in these people. The finding of poor anti-spike antibody responses in organ transplant recipients following the first dose of mRNA vaccines indicates that these patients are still at a higher early risk of COVID-19 despite vaccination. Deep immunophenotyping of transplant recipients with vaccination, including characterization of memory B-cell and T-cell responses, is significant in deciding vaccination strategies and immunologic responses following the second dose [40].

In December 2020, the Canadian National Advisory Committee on Immunization recommended that a COVID-19 vaccine should not be provided to breastfeeding or pregnant women until further evidence is available; however, it can be considered in special scenarios where benefits outweigh risks. On the contrary, guidance from specialist bodies in the US is against withholding the COVID-19 vaccination from breastfeeding or pregnant women [41].

\section{An Overview of Herd Immunity and the Reason for Mass Vaccination}

Herd immunity, achieved either by vaccination or natural infection, is pursued to stop the disease spread and control the pandemic; but mounting evidence suggests that natural infection is not able to achieve herd immunity as expected. Some reinfections with SARS-CoV-2 have been reported, indicating that immunological memory generated by natural infection may not strong or last long enough to protect people from reinfection [30]. Recommendations from public health specialists include giving at least one dose of vaccine to previously infected patients to reinforce their immunity. However, it is highly unrecommended to give dual-dose vaccinations to individuals who were previously infected with COVID-19. Overall, natural immunity (i.e., immunity received by recovering from the virus) typically fosters a robust immune response against SARS-CoV-2 and its variants. It is important to guarantee high vaccine uptake and herd immunity in the population. The threshold of herd immunity for SARS-CoV-2 is projected at $60-83 \%$ [42].

Achieving enough vaccine coverage in workingage adults is vital if herd immunity is a goal. Information highlighting benefits of herd immunity may help reduce the vaccine hesitancy of COVID-19 [43]. Herd immunity can be achieved only by mass vaccination. Vaccine hesitancy is the main barrier to accomplishing herd immunity [44]. A widespread belief that vaccination is riskier than COVID-19 itself is an obstruct to herd immunity. Transparency is perhaps the most important concept when dealing with public health, the pandemic, and individual healthcare. This kind of belief will prolong the pandemic [45]. If substantial immune evasion occurs (due to variants), available vaccines may still have some efficacy on individuals; but they might

Citation: Cheryl Ann Alexander1and Lidong Wang2* COVID-19, the Pandemic, and Challenges Associated with Vaccines Op Acc J Bio Sci \& Res 9(1)-2021 
induce viral selection and escape at the population level, making fulfilling herd immunity difficult [31].

\section{Vaccine Hesitancy}

Vaccine hesitancy refers to the delay in acceptance or refusal of vaccines though vaccines and services are available. It is complex and context specific, being associated with time, place, and vaccines. It is also a complicated public health issue regarding efficacy, safety, or a need for vaccination. It has depolarized vaccine-supporters and their anti-vaccine counterparts. It is individual's behavior and affected by some factors such as knowledge and experiences in the past. It is also influenced by factors such as convenience, complacency, and confidence. Specifically, factors resulting in vaccine hesitancy include 1) having difficulties in accessing vaccines, 2 ) not realizing a need for a vaccine (such as the under-estimation of the severity of COVID-19), and 3) lacking confidence in or being fearful of vaccines. A stronger intention of COVID-19 vaccination is related to more collective responsibility, less complacency, more confidence, and a younger age. Much work stress also causes demands for the vaccination $[46,47]$. Trust in doctors (recommending and administering vaccines), healthcare system, and vaccine information obtained via the media plays a significant role in the decision-making of vaccination. However, misinformation regarding COVID-19 vaccine on major social media companies has been a problem, especially for newly available vaccines. The Strategic Advisory Group of Experts on Immunization Working Group insisted that healthcare professionals' poor communication played a significant role in the vaccine hesitancy [48].

Misinformation and vaccine hesitancy are barriers to achieving coverage and community immunity. Misinformation spread via multiple channels has negative effects on the COVID-19 vaccine acceptance. Accurate, clear communication and Transparent, evidence-informed policies are important for all relevant stakeholders. A higher level of trust in information from government sources facilitates the vaccine acceptance; an accelerated pace of vaccination possibly brings up public anxieties and compromises the acceptance. Government officials need to have consistent and clear communications on vaccines, which is key to creating public confidence in vaccine programs such as introducing how vaccines are developed and how they work; explaining the effectiveness of a vaccine, the time needed for protection, and the implication of population-wide coverage to achieve community immunity. Respected groups based on community and nongovernmental organizations, for example, the Red Cross, also assist building trust in COVID-19 vaccines. People with a better income are more likely accept vaccines than people with a lower income. Men are generally less likely to accept vaccines or their employers' suggestion on vaccination. An older person is more likely to take a vaccine [49].

Although vaccination emergency is somewhat regarded as a mandatory commitment to reduce infectious contacts and hospitalization, vaccination hesitancy is still a concern, especially for the messenger RNA (mRNA)-based vaccines. Correct information on mRNA-based vaccines was ever very limited in Italy. Some news reached a wide community due to commercial impact, leading to wrong expectations. The purported $95 \%$ efficiency of mRNA vaccines was intensely criticized. Misleading information regarding RNA-based vaccines caused vaccine hesitancy with a high degree in Italy and the US. The complicated issue of vaccinology (especially during a pandemic) should be a basic educational mission for public health [50].

Features of COVID-19 vaccines might change over time (e.g., technology used, efficacy, country of manufacturer, effectiveness against variants, risk of severe side-effects, and post-vaccination transmission as more evidence emerges). Vaccine hesitancy is related to the potential vaccine features. Most people are not likely completely for or against COVID-19 vaccines. Depending on their profiles, preferences, and features of available vaccines, vaccinehesitant persons may consider taking a vaccine or delay to obtain another vaccine [43]. The Anti-vaccination behavior of COVID-19 is significantly related to some situations: lower educational level, female gender, no chronic condition report, ages with an inverted U-shaped relationship, lower perceived severity of COVID-19 infection, poorer compliance with suggested vaccination in the past, etc. Vaccine acceptance in the working-age people relies on the features of new vaccines and the nationwide vaccination strategy [44].

The vaccine hesitancy of the general public is mainly due to indifference to COVID-19 infection risks, misconceptions about contracting SARS-CoV-2 from vaccines, and concerns about possible vaccine side effects. Although immigrant families have positive attitudes on vaccines, their vaccination rate was low due to various reasons such as immigration status, language differences, transportation problems poor local availability of vaccines, low health literacy, lack of health insurance, and vaccine costs. Whether insured, uninsured, or underinsured, many people in the racial/ ethnic minority community do not have a medical home or a regular primary care provider, which limits their access to vaccines. In addition, underrepresented groups in the US are often more skeptical of the efficacy and safety of new medical products [51]. 
Vaccine hesitancy is characterized by uncertainty and ambivalence about vaccination. Surveys indicated many people with vaccine hesitancy are from ethnic minorities. A UK survey in December 2020 showed that vaccine hesitancy was higher among black, Bangladeshi, and Pakistani people compared with white people. Population groups with frequent address change have lower vaccination rates which is common among people from ethnic minorities and makes NHS records inaccurate [52]. Concerns about vaccine hesitancy have been laid primarily at the feet of African American and Latinx communities in the US. Disinformation and fears of vaccine consequences have spread through Latinx communities. Some news media's focus on mistrust or seemingly ridiculous conspiracies ignored the racist structures and led to health disparities [45]. Some Indigenous and African people are hesitant in vaccination due to long-standing racism and abuse [41].

\section{Conclusion}

The advantages of mRNA vaccines over conventional vaccines lie in the capability of quick development and manufacturing at low cost, with good efficacy, etc. The mRNA-1273 vaccine and BNT162b2 vaccine have high degree of efficacy and immunogenicity. There is a reduction of asymptomatic cases in vaccinated individuals. There is a risk of infection with a variant virus even if individuals have completed vaccination; some individuals, however, may be susceptible to greater side effects from vaccination against the pandemic, have chronic illnesses that predispose them to increased side effects, or simply not trust the methods behind the vaccine development because transparency has not been the main method of finding out information regarding the vaccines. Furthermore, some individuals may suffer increased side effects resulting in death or disability.

The probability of the COVID-19 infection is higher in individuals with older ages, major baseline disability, and cardiovascular or pulmonary comorbidities. Yet, the number one comorbidity for adults is obesity. Natural infection does not enable herd immunity as expected. Vaccine hesitancy is a major barrier to accomplishing herd immunity. Concerns about vaccine side effects, indifference to COVID-19 infection risks, and misconceptions about contracting SARSCoV-2 from vaccines are major reasons of the COVID-19 vaccine hesitancy of the general public.

\section{Acknowledgment}

Authors thank Technology \& Healthcare Solutions, Mississippi, USA for support.

\section{References}

1. Swift MD, Breeher LE, Tande AJ, Tommaso CP, Hainy CM, Chu H, Murad MH, Berbari EF, Virk A (2021) Effectiveness of mRNA COVID-19 vaccines against SARS-CoV-2 infection in a cohort of healthcare personnel. Clinical Infectious Diseases: An Official Publication of the Infectious Diseases Society of America. Apr 26.

2. Oliver SE, Gargano JW, Scobie H, Wallace M, Hadler SC, et al. (2021) The advisory committee on immunization practices' interim recommendation for use of Janssen COVID-19 vaccine-United States, February 2021. Morbidity and Mortality Weekly Report. Mar 5; 70 (9): 329.

3. Khodavirdipour A, Jabbari S, Keramat F, Alikhani MY (2021) Concise Update on Genomics of COVID-19; In Approach to Its latest Mutations, Escalated Contagiousness, and Vaccine Resistance. Global Medical Genetics. Mar 15.

4. Wise J (2021) Covid-19: Is the UK heading towards mandatory vaccination of healthcare workers?

5. Padma TV (2021) India's COVID-vaccine woes-by the numbers. Nature, 592(7855): 500-501.

6. Guidry JP, Perrin PB, Laestadius LI, Vraga EK, Miller CA, Fuemmeler BF, ... \& Carlyle KE (2021) US public support for COVID-19 vaccine donation to low-and middle-income countries during the COVID-19 pandemic. Vaccine, 39(17): 2452-2457.

7. Eisenstadt M, Ramachandran M, Chowdhury N, Third A, \& Domingue J (2020) COVID-19 antibody test/vaccination certification: there's an app for that. IEEE Open Journal of Engineering in Medicine and Biology, 1: 148-155.

8. Health TLP (2021) COVID-19 in France: challenges and opportunities. The Lancet. Public Health, 6(4): e192.

9. Mertz L (2021) New Vaccine-Manufacturing Methods Are Moving Away from the Egg. IEEE pulse, 12(1): 7-11.

10. Zhang Y, Li D, Zhao H, Wang L, Liao Y, Li X, Li Q (2021) The role of multiple SARS-CoV-2 viral antigens in a vaccine-induced integrated immune response. Vaccine.

11. Corey L, Mascola JR, Fauci AS, \& Collins FS (2020) A strategic approach to COVID-19 vaccine R\&D. Science. 368(6494): 948-950.

12. Andreadakis Z, Kumar A, Román RG, Tollefsen S, Saville M, \& Mayhew S (2020) The COVID-19 vaccine development landscape. Nature reviews. Drug discovery, 19(5): 305-306.

13. Teo SP (2021) Review of COVID-19 Vaccines and Their Evidence in Older Adults. Annals of geriatric medicine and research. Mar; 25(1): 4.

14. Goldschmidt K (2021) COVID-19 vaccines for children: The essential role of the pediatric nurse. Journal of Pediatric Nursing.

15. Vitiello A \& Ferrara F (2021) Brief review of the mRNA vaccines COVID-19. Inflammopharmacology, 1-5.

16. Gibbs WW (2021) This is how to vaccinate the world, Spectrum, IEEE, 32-37.

17. Negahdaripour M (2021) COVID-19 Vaccine Global Access Is an Urgency. Iranian Journal of Medical Sciences, 46(2): 79-80.

18. Gurajala S (2021) A Sneak Peek into COVID-19 Vaccines-Present Status. Journal of Clinical \& Diagnostic Research, 15(3).

19. Tauh T, Mozel M, Meyler P, Lee SM, MAS M (2021) What is the evidence for extending the SARS-CoV-2 (COVID-19) vaccine dosing schedule? Fertility treatment options after vasectomy. March: 67.

20. Logunov DY, Dolzhikova IV, Shcheblyakov DV, Tukhvatulin AI, Zubkova OV, et al. (2021) Safety and efficacy of an rAd26 and rAd5 vector-based heterologous prime-boost COVID-19 vaccine: an interim analysis of a randomised controlled phase 3 trial in Russia. The Lancet. 397(10275): 671-681.

21. Polack FP, Thomas SJ, Kitchin N, Absalon J, Gurtman A, et al. (2020) Safety and efficacy of the BNT162b2 mRNA Covid-19 vaccine. New England Journal of Medicine. Dec 10.

22. Olliaro P (2021) What does 95\% COVID-19 vaccine efficacy really mean? The Lancet Infectious Diseases. Jun 1; 21(6): 769.

23. Altmann DM, Boyton RJ \& Beale R (2021) Immunity to SARS-CoV-2

Citation: Cheryl Ann Alexander1and Lidong Wang2* COVID-19, the Pandemic, and Challenges Associated with Vaccines Op Acc J Bio Sci \& Res 9(1)-2021 
variants of concern. Science, 371(6534): 1103-1104.

24. McEllistrem MC, Clancy CJ, Buehrle DJ, Lucas A, Decker BK (2021) Single dose of a mRNA SARS-CoV-2 vaccine is associated with lower nasopharyngeal viral load among nursing home residents with asymptomatic COVID-19. Clinical Infectious Diseases. Mar 26.

25. Iacobucci G (2021) Covid-19: Infections fell by $65 \%$ after first dose of AstraZeneca or Pfizer vaccine, data show. BMJ. Apr 23; 373: n1068.

26. Hacisuleyman E, Hale C, Saito Y, Blachere NE, Bergh M, et al. (2021) Vaccine breakthrough infections with SARS-CoV-2 variants. New England Journal of Medicine. Jun 10; 384(23): 2212-2218.

27. Zou J, Xie X, Fontes-Garfias CR, Swanson KA, Kanevsky I, et al. (2021) The effect of SARS-CoV-2 D614G mutation on BNT162b2 vaccineelicited neutralization. npj Vaccines. Mar 25; 6(1): 1-4.

28. McCormick KD, Jacobs JL \& Mellors JW (2021) The emerging plasticity of SARS-CoV-2. Science, 371(6536): 1306-1308.

29.

30. Muik A, Wallisch AK, Sänger B, Swanson KA, Mühl J, et al. (2021) Neutralization of SARS-CoV-2 lineage B. 1.1. 7 pseudovirus by BNT162b2 vaccine-elicited human sera. Science. 371(6534): 11521153.

31. Chen J, Lu H (2021) New challenges to fighting COVID-19: Virus variants, potential vaccines, and development of antivirals. Bioscience trends.

32. Atlani-Duault L, Lina B, Chauvin F, Delfraissy JF, Malvy D (2021) Immune evasion means we need a new COVID-19 social contract. The Lancet Public Health. Apr 1; 6(4): e199-200.

33. Li M, Lou F, \& Fan H (2021) SARS-CoV-2 variants: a new challenge to convalescent serum and mRNA vaccine neutralization efficiency. Signal Transduction and Targeted Therapy, 6(1): 1-3.

34. Gee J, Marquez P, Su J, Calvert GM, Liu R, et al. (2021) First month of COVID-19 vaccine safety monitoring-United States, December 14, 2020-January 13, 2021. Morbidity and Mortality Weekly Report. Feb 26; 70(8): 283.

35. Ledford $H$ (2021). COVID vaccnes and blood clots: five key questions. Nature, 592(7855): 495-496.

36. COVID C, Team R (2020) Allergic reactions including anaphylaxis after receipt of the first dose of Pfizer-BioNTech COVID-19 vaccine-United States, December 14-23, 2021. Morbidity and Mortality Weekly Report. Jan 15; 70(2): 46.

37. Wei N, Fishman M, Wattenberg D, Gordon M, \& Lebwohl M (2021) "COVID arm": A reaction to the Moderna vaccine. JAAD case reports.

38. Campos-Outcalt D (2021) ACIP recommendations for COVID-19 vaccines-and more. The Journal of Family Practice, 70(2): 86-89.

39. Seachrist EJ (2021) Multiple sclerosis, B cell therapy, and the COVID-19 vaccine. Eneurologicalsci, 22: 100319.

40. Ammirati E, Cavalotti C, Milazzo A, Pedrotti P, Soriano F, et al. (2021)
Temporal relation between second dose BNT162b2 mRNA Covid-19 vaccine and cardiac involvement in a patient with previous SARS-COV-2 infection. International journal of cardiology. Heart \& vasculature. Jun 1.

41. Boyarsky BJ, Werbel WA, Avery RK, Tobian AA, Massie AB, et al. (2021) Immunogenicity of a single dose of SARS-CoV-2 messenger RNA vaccine in solid organ transplant recipients. Jama. May 4; 325(17): 1784-1786.

42. Zipursky JS, Greenberg RA, Maxwell C, Bogler T (2021) Pregnancy, breastfeeding and the SARS-CoV-2 vaccine: an ethics-based framework for shared decision-making. CMAJ, 193(9): E312-E314.

43. Alkandari D, Herbert JA, Alkhalaf MA, Yates C, Panagiotou S (2021) SARS-CoV-2 vaccines: fast track versus efficacy. The Lancet Microbe. Mar 1; 2(3): e89-90.

44. Verger P, Peretti-Watel P (2021) Understanding the determinants of acceptance of COVID-19 vaccines: a challenge in a fast-moving situation. The Lancet Public Health, 6(4): e195-e196.

45. Schwarzinger M, Watson V, Arwidson P, Alla F, Luchini S (2021) COVID-19 vaccine hesitancy in a representative working-age population in France: a survey experiment based on vaccine characteristics. The Lancet Public Health, 6(4): e210-e221.

46. Reverby SM (2021) Racism, disease, and vaccine refusal: People of color are dying for access to COVID-19 vaccines. PLoS Biology, 19(3): e3001167.

47. Kwok KO, Li KK, Wei WI, Tang A, Wong SY, Lee SS (2021) Influenza vaccine uptake, COVID-19 vaccination intention and vaccine hesitancy among nurses: A survey. International journal of nursing studies. 114: 103854.

48. Detoc M, Bruel S, Frappe P, Tardy B, Botelho-Nevers E, et al. (2020) Intention to participate in a COVID-19 vaccine clinical trial and to get vaccinated against COVID-19 in France during the pandemic. Vaccine, 38(45): 7002-7006.

49. Diem L, Friedli C, Chan A, Salmen A, Hoepner R (2021) Vaccine hesitancy in patients with multiple sclerosis: preparing for the SARS-CoV-2 vaccination challenge. Neurology-Neuroimmunology Neuroinflammation. 8(3).

50. Lazarus JV, Ratzan SC, Palayew A, Gostin LO, Larson HJ, et al. (2021) A global survey of potential acceptance of a COVID-19 vaccine. Nature medicine. 27(2): 225-228.

51. Chirumbolo S (2021) Vaccination hesitancy and the "myth" on mRNAbased vaccines in Italy in the COVID-19 era: Does urgency meet major safety criteria? Journal of Medical Virology 93(7): 4049-4053.

52. Clark EH, Fredricks K, Woc-Colburn L, Bottazzi ME, Weatherhead J (2021) Preparing for SARS-CoV-2 vaccines in US immigrant communities: strategies for allocation, distribution, and communication.

53. Razai MS, Osama T, McKechnie DG, Majeed A (2021) Covid-19 vaccine hesitancy among ethnic minority groups.

\footnotetext{
*Corresponding author: Lidong Wang, Email: lidong@iser.msstate.edu
}

\author{
Next Submission with BGSR follows: \\ - Rapid Peer Review \\ - Reprints for Original Copy \\ - E-Prints Availability \\ - Below URL for auxiliary Submission Link: https://biogenericpublishers.com/submit-manuscript/
}

